\title{
SUPRATHERMAL ELECTRON STRAHL WIDTHS IN THE PRESENCE OF NARROW-BAND WHISTLER WAVES IN THE SOLAR WIND
}

\author{
P. Kajdič ${ }^{1}$, O. Alexandrova ${ }^{2}$, M. Maksimovic ${ }^{2}$, C. Lacombe $^{2}$, and A. N. FazaKerley ${ }^{3}$ \\ ${ }^{1}$ Instituto de Geofísica, Universidad Nacional Autónoma de México, Mexico City, Mexico; primoz@geofisica.unam.mx \\ ${ }^{2}$ LESIA, Observatoire de Paris, PSL Research University, CNRS, UPMC UniversitéParis 06, Université Paris-Diderot, 5 Place Jules Janssen, \\ F-92190 Meudon, France \\ ${ }^{3}$ Mullard Space Science Laboratory, University College London, UK \\ Received 2016 June 17; revised 2016 September 12; accepted 2016 September 16; published 2016 December 15
}

\begin{abstract}
We perform the first statistical study of the effects of the interaction of suprathermal electrons with narrow-band whistler mode waves in the solar wind (SW). We show that this interaction does occur and that it is associated with enhanced widths of the so-called strahl component. The latter is directed along the interplanetary magnetic field away from the Sun. We do the study by comparing the strahl pitch angle widths in the SW at $1 \mathrm{AU}$ in the absence of large scale discontinuities and transient structures, such as interplanetary shocks, interplanetary coronal mass ejections, stream interaction regions, etc. during times when the whistler mode waves were present and when they were absent. This is done by using the data from two Cluster instruments: Spatio Temporal Analysis of Field Fluctuations experiment (STAFF) data in the frequency range between $\sim 0.1$ and $\sim 200 \mathrm{~Hz}$ were used for determining the wave properties and Plasma Electron And Current Experiment (PEACE) data sets at 12 central energies between $\sim 57 \mathrm{eV}$ (equivalent to $\sim 10$ typical electron thermal energies in the $\mathrm{SW}, E_{T}$ ) and $\sim 676 \mathrm{eV}$ $\left(\sim 113 E_{T}\right)$ for pitch angle measurements. Statistical analysis shows that, during the intervals with the whistler waves, the strahl component on average exhibits pitch angle widths between $2^{\circ}$ and $12^{\circ}$ larger than during the intervals when these waves are not present. The largest difference is obtained for the electron central energy of $\sim 344 \mathrm{eV}\left(\sim 57 E_{T}\right)$.
\end{abstract}

Key words: acceleration of particles - scattering - solar wind - turbulence - waves

\section{INTRODUCTION}

It was discovered very early that the electron velocity distribution function (VDF) in the solar wind (SW) is composed of different components (e.g., Montgomery et al. 1968; Feldman et al. 1975, 1978; Rosenbauer et al. 1976; Lin 1998; Maksimovic et al. 2005). About 95\% of all the electrons belong to the thermal core population with typical temperature of $\sim 10 \mathrm{eV}$. These electrons are marginally collisional at $1 \mathrm{AU}$ (Phillips \& Gosling 1990) and their VDF can be described as bi-Maxwellian (one in parallel and one in perpendicular direction, with respect to the mean field).

Electrons with energies between $\sim 70 \mathrm{eV}$ and $\sim 2 \mathrm{keV}$ are referred to as suprathermal. These are collisionless at $1 \mathrm{AU}$ (Scudder \& Olbert 1979; Fairfield \& Scudder 1985; Ogilvie et al. 2000), so they are not in thermodynamic equilibrium. Suprathermal electrons are composed of two components: halo exhibits approximately isotropic VDF with suprathermal tails that can be approximated by a bi-kappa distribution (e.g., Maksimovic et al. 1997, 2005; Štverák et al. 2009). The strahl component can be described as field aligned antisunward directed beams of electrons. Finally, the electrons with energies above $2 \mathrm{keV}$ compose isotropic superhalos (Lin 1998).

There has been some discussion about the origin of suprathermal electrons. Pierrard et al. (1999) used the Wind observations of the electron VDF at $1 \mathrm{AU}$ to derive the coronal VDF and concluded that suprathermal electrons must already be present in the corona. Vocks \& Mann (2003), Vocks et al. (2008) and Vocks (2012) postulate that the suprathermal population is formed in the inner corona by resonant interaction with antisunward propagating whistler waves. These waves scatter the sunward propagating portion of core electrons from small velocities parallel to magnetic field $\left(v_{\|}\right)$to large perpendicular velocities $\left(v_{\perp}\right)$, thereby forming the halo.

Whatever the origin, the antisunward propagating suprathermal electrons (in the plasma frame) are subject to focusing effects by the diverging interplanetary magnetic field (IMF), due to conservation of the particle's magnetic moment (e.g., Owens et al. 2008). If no other effects were present in the interplanetary (IP) space, these electrons would be focused into a very narrow beam or strahl. Observations, however, show strahl with a finite width (e.g., Fitzenreiter et al. 1998). Hence, some mechanism(s) must exist in the IP space which scatter the strahl electrons toward larger pitchangles (PA).

It is commonly postulated that a halo at large heliocentric distances is formed by scattering of the strahl electrons. Some indirect evidence points in this direction: Maksimovic et al. (2005) and Štverák et al. (2009), for example, have shown that while the core fractional density remains constant with the distance from the Sun, the halo and the strahl fractional densities vary in opposite ways. The halo fractional density increases with increasing heliocentric distance, that of the strahl decreases, while their sum remains roughly constant.

Electromagnetic fluctuations (frequency $\omega$ ) can resonantly interact with electrons in the SW if their Doppler shifted frequency in the electron frame is equal to a multiple of the electron cyclotron frequency $\Omega_{e}$. This resonance condition reads:

$$
\omega-k_{\|} v_{\|}=n \Omega_{e} ; n=1,2,3, \ldots,
$$

where $k_{\|}$and $v_{\|}$are the components of the wave vector and electron velocity parallel to the background magnetic field. 
Whistler waves, which have frequencies $\omega<\Omega_{e}$ and a righthanded polarization with respect to the background magnetic field (e.g., Gurnett \& Bhattacharjee 2005), can resonate with electrons if $k_{\|} \cdot v_{\|}$is negative: antisunward propagating electrons can only interact with sunward propagating whistler waves.

Two potential sources of whistler mode fluctuations in the SW are (e.g., Saito \& Gary 2007) wave-particle interactions and wave-wave interactions. The first can generate whistler fluctuations through electromagnetic instabilities, such as heat flux instability, and the electron temperature anisotropy instability. The wave-wave interactions may result in magnetic fluctuations cascading. It is well known that at low frequencies the magnetic power spectrum in the SW exhibits frequency dependence $f^{-5 / 3}$, (e.g., Bruno \& Carbone 2013). At around the proton cyclotron frequency the spectrum becomes steeper (e.g., see the review of Alexandrova et al. 2013). The nature of this small scale turbulent cascade is still an open question. Some authors, e.g., (Denskat et al. 1983; Ghosh et al. 1996; Stawicki et al. 2001; Smith et al. 2006a), suggest that fluctuations in this range may be whistler mode waves with broad spectrum (as opposed to narrow-band whistler wave modes described here in this paper).

Broadband whistler waves propagating parallel to the background $B$-field were introduced in simulations by Vocks et al. (2005), who showed that in IP space these waves could indeed disperse the strahl. Pierrard et al. (2011) also proposed that the strahl electrons could be scattered off the whistler broadband turbulence with wave vectors parallel to the background magnetic field. However, observations show that within this small scale range, turbulent fluctuations are dominated by quasi-perpendicular wave vectors $k_{\perp} \gg k_{\|}$ (Mangeney et al. 2006; Alexandrova et al. 2008; Chen et al. 2010; Sahraoui et al. 2010; Roberts et al. 2013) and not by quasi-parallel ones as needed in the previously mentioned models (see discussion in Section 4 for more details). Alternatively, Pavan et al. (2013) suggested that selfgenerated Langmuir waves at plasma frequency could also scatter the strahl in pitch angle and energy, resulting in significant broadening of its VDF.

Direct observations of halo formation from the strahl component have been reported by Gurgiolo et al. (2012). These authors examined electron VDFs obtained by the Plasma Electron And Current Experiment (PEACE) instrument on board the Cluster spacecraft. Gurgiolo et al. (2012) show a handful of time intervals during which scattering of the strahl, into what they call the proto-halo, and then into the halo, was observed. This occurred for electrons at energies $\lesssim 50 \mathrm{eV}$ during time intervals of $\sim 10 \mathrm{~s}$. The authors also examined magnetic field turbulence from the Spatio Temporal Analysis of Field Fluctuations experiment (STAFF) and Fluxgate Magnetometer (FGM) data sets and concluded that no monochromatic whistler mode waves were present during the examined intervals, but that there were enhanced levels of broadband turbulence.

In contrast to previous works, we study the broadening of the strahl during times when narrow-band whistler waves are present in the SW. By narrow-band we mean that in the spectra of magnetic field turbulence, these waves produce a clear distinct bump, which is superimposed on the spectra of permanent background turbulence. Recently, Lacombe et al. (2014) performed a study of such waves and determined preferential conditions in the SW for their observations. These include a low level of background turbulence, a slow wind, a relatively large electron heat flux, and a low electron collision frequency. The authors related the presence of the whistlers preferentially to the whistler heat flux instability and in rare cases to the anisotropy instability of the total electron temperature.

This paper is organized in the following manner: in Section 2 we describe the instruments and the data sets used in this work and present a case study. In Section 3 we discuss the properties of the IMF and the SW during time intervals in our sample, the observational properties of the whistler waves and the measured strahl widths. In Section 4 we discuss the results and summarize them.

\section{OBSERVATIONS}

\subsection{Instruments and Data sets}

The Cluster mission consists of four identical spacecraft in orbit around the Earth. It provides magnetic field and plasma measurements in the near-Earth environment. The satellites carry several instruments on board. Here, we use the data provided by five instruments: the FGM (Balogh et al. 2001), the Cluster Ion Spectrometer (CIS, Rème et al. 2001), the PEACE (Johnstone et al. 1997), the STAFF (CornilleauWehrlin et al. 1997, 2003) and the Waves of High frequency and Sounder for Probing of Electron density by Relaxation (WHISPER, Décréau et al. 1997).

All the data used in this work were obtained from the Cluster Science Archive (http://www.cosmos.esa.int/web/csa), which is maintained by the European Space Agency. We use the FGM magnetic field vectors and the CIS-HIA SW ion moments with 0.2 and $4 \mathrm{~s}$ time resolution, respectively. To obtain electron pitch angle distributions we use PEACE PITCH_SPIN data sets. These contain data from both PEACE sensors, namely the High Energy Electron Analyser and the Low Energy Electron Analyser. The data in them are binned in twelve $15^{\circ}$ pitch angle bins and 44 energy bins. In our work, we use approximate central energies of 676, 536, 430, 344, 276, 220, 175, 140, 111, 89,71 , and $57 \mathrm{eV}$. We note here, that the energies in PITCH_SPIN data sets are not corrected for spacecraft potential, however, during our intervals, the potential was typically less than $5 \mathrm{~V}$, which is far less than the energy intervals used here. The central energies between 57 and $676 \mathrm{eV}$ were chosen since we find that at higher energies the PADs become too noisy and not many good examples could be obtained. The lower threshold was chosen since the usual break point between the core and the suprathermal electrons is around $60 \mathrm{eV}$ (Feldman et al. 1975). PEACE data are available in $4 \mathrm{~s}$ time resolution. We use WHISPER data in order to make sure that the Cluster probes are not located inside the Earth's foreshock. This is done by checking for the presence of the electrostatic or Langmuir waves which are commonly present in the foreshock.

The STAFF experiment measures the three orthogonal components of the magnetic field fluctuations. It comprises two onboard analyzers: the wave form unit (STAFF-SC) provides digitized wave forms up to either $12.5 \mathrm{~Hz}$ or $180 \mathrm{~Hz}$, depending on the spacecraft telemetry rate. The Spectrum Analyzer (STAFF-SA) uses the three magnetic field and two electric field components (from the EFW experiment, 
Table 1

Sampled Time Intervals

\begin{tabular}{|c|c|c|c|c|c|}
\hline \multicolumn{3}{|c|}{ With Whistlers } & \multicolumn{3}{|c|}{ Without Whistlers } \\
\hline $\begin{array}{l}\text { Date } \\
\text { YYYY MM DD }\end{array}$ & $\begin{array}{l}\text { Time UT } \\
\text { hr:mn }\end{array}$ & $\begin{array}{c}\text { Spacecraft } \\
\text { STAFF, FGM, DEF/Ion moments }\end{array}$ & $\begin{array}{c}\text { Date } \\
\text { YYYY/MM/DD }\end{array}$ & $\begin{array}{l}\text { Time UT } \\
\text { hr:mn }\end{array}$ & $\begin{array}{c}\text { Spacecraft } \\
\text { STAFF, IMF, DEF/Ion moments }\end{array}$ \\
\hline $2001 \mathrm{Feb} 19$ & $17: 15-17: 19$ & $C 1 / C 1$ & $2001 \mathrm{Feb} 19$ & $19: 22-19: 29$ & $C 1 / C 1$ \\
\hline 2001 Feb 19 & $17: 21-17: 42$ & $C 1 / C 1$ & 2001 Feb 19 & $19: 55-20: 10$ & $C 1 / C 1$ \\
\hline 2002 Feb 09 & $02: 30-02: 40$ & $C 1 / C 1$ & 2002 Feb 09 & $02: 10-02: 20$ & $C 1 / C 1$ \\
\hline 2003 Jan 30 & $00: 38-01: 35$ & $C 1 / C 1$ & 2003 Jan 30 & 00:00-00:30 & $\mathrm{Cl} / \mathrm{Cl}$ \\
\hline 2003 Jan 30 & $02: 42-03: 10$ & $C 1 / C 1$ & 2003 Jan 30 & $03: 25-03: 35$ & $\mathrm{Cl} / \mathrm{Cl}$ \\
\hline 2003 Jan 30 & $07: 05-07: 33$ & $C 1 / C 1$ & 2003 Jan 30 & $08: 16-08: 18$ & $C 1 / C 1$ \\
\hline 2003 Jan 30 & $07: 38-07: 45$ & $C 1 / C 1$ & 2003 Jan 30 & $09: 15-09: 25$ & $C 1 / C 1$ \\
\hline 2004 Feb 09 & $19: 19-19: 23$ & $C 4 / C 1$ & 2004 Feb 09 & $19: 12-19: 17$ & $C 4 / C 1$ \\
\hline 2004 Feb 09 & $20: 36-20: 43$ & $C 4 / C 1$ & 2004 Feb 09 & 19:50-20:00 & $C 4 / C 1$ \\
\hline 2004 Feb 09 & $20: 59-21: 02$ & $C 4 / C 1$ & 2004 Feb 09 & $20: 20-20: 30$ & $C 4 / C 1$ \\
\hline 2004 Feb 09 & $22: 04-22: 08$ & $C 4 / C 1$ & 2004 Feb 09 & $21: 50-22: 00$ & $C 4 / C 1$ \\
\hline 2004 Feb 09 & $22: 44-22: 45$ & $C 4 / C 1$ & 2004 Feb 09 & $22: 50-23: 00$ & $C 4 / C 1$ \\
\hline 2004 Apr 18 & $11: 40-12: 15$ & $C 1 / C 1$ & 2005 Feb 16 & $11: 50-12: 00$ & $C 1 / C 1$ \\
\hline 2005 Feb 16 & $11: 19-11: 22$ & $C 1 / C 1$ & 2007 Mar 04 & 07:00-07:10 & $C 1 / C 1$ \\
\hline 2007 Mar 04 & $07: 18-07: 26$ & $C 1 / C 1$ & 2009 Feb 08 & 04:00-04:30 & $C 2 / C 1$ \\
\hline 2009 Feb 08 & $04: 33-06: 18$ & $C 2 / C 1$ & 2009 Feb 20 & 03:00-03:10 & $C 2 / C 1$ \\
\hline 2009 Feb 20 & $03: 38-03: 48$ & $C 2 / C 1$ & 2009 Feb 20 & $04: 50-05: 00$ & $C 2 / C 1$ \\
\hline 2009 Feb 20 & $03: 48-04: 05$ & $C 2 / C 1$ & 2009 Feb 21 & $14: 50-15: 00$ & $C 2 / C 1$ \\
\hline 2009 Feb 21 & $14: 10-14: 39$ & $C 2 / C 1$ & 2009 Feb 21 & $15: 45-15: 55$ & $C 2 / C 1$ \\
\hline 2009 Feb 21 & $15: 20-15: 27$ & $C 2 / C 1$ & 2009 Apr 27 & $04: 40-04: 50$ & $C 2 / C 1$ \\
\hline 2009 Apr 27 & $04: 53-05: 21$ & $C 2 / C 1$ & 2010 Jan 11 & $20: 00-20: 10$ & $C 2 / C 1$ \\
\hline 2010 Jan 11 & $20: 17-20: 21$ & $C 2 / C 1$ & 2010 Jan 11 & $20: 30-20: 38$ & $C 2 / C 1$ \\
\hline 2010 Jan 11 & $20: 25-20: 27$ & $C 2 / C 1$ & 2010 Feb 23 & $17: 13-17: 20$ & $C 2 / C 1$ \\
\hline 2010 Feb 23 & $17: 03-17: 13$ & $C 2 / C 1$ & 2010 Feb 25 & $14: 25-14: 35$ & $C 2 / C 1$ \\
\hline 2010 Feb 25 & $13: 37-13: 57$ & $C 2 / C 1$ & 2010 Feb 25 & $20: 58-21: 03$ & $C 2 / C 1$ \\
\hline 2010 Feb 25 & $20: 10-20: 58$ & $C 2 / C 1$ & 2010 Feb 28 & $11: 15-11: 25$ & $C 2 / C 1$ \\
\hline 2010 Feb 25 & $21: 09-21: 47$ & $C 2 / C 1$ & 2010 Mar 15 & $22: 40-22: 50$ & $C 2 / C 1$ \\
\hline 2010 Feb 28 & 11:04-11:09 & $C 2 / C 1$ & 2010 Mar 15 & $23: 18-23: 20$ & $C 2 / C 1$ \\
\hline 2010 Mar 15 & $23: 03-23: 05$ & $C 2 / C 1$ & 2010 Mar 16 & 01:35-01:39 & $C 2 / C 1$ \\
\hline 2010 Mar 15 & $23: 26-23: 37$ & $C 2 / C 1$ & 2010 Apr 18 & 20:01-20:07 & $C 2 / C 1$ \\
\hline 2010 Mar 15 & $23: 40-23: 55$ & $C 2 / C 1$ & 2010 Apr 19 & $04: 45-04: 55$ & $C 2 / C 1$ \\
\hline 2010 Mar 15 & $23: 58-24: 00$ & $C 2 / C 1$ & & & \\
\hline 2010 Mar 16 & $00: 00-00: 23$ & $C 2 / C 1$ & & & \\
\hline 2010 Mar 16 & $00: 29-00: 38$ & $C 2 / C 1$ & & & \\
\hline 2010 Mar 16 & 01:29-01:34 & $C 2 / C 1$ & & & \\
\hline 2010 Apr 18 & $19: 40-19: 54$ & $C 2 / C 1$ & & & \\
\hline 2010 Apr 19 & $04: 10-04: 18$ & $C 2 / C 1$ & & & \\
\hline
\end{tabular}

Gustafsson et al. 1997) to build a $5 \times 5$ spectral matrix, which in normal telemetry rate has a time resolution of $4 \mathrm{~s}$ and the frequency range between $8 \mathrm{~Hz}$ and $4 \mathrm{kHz}$. The two analyzers provide the sense of polarization, the ellipticity and the propagation direction of the observed fluctuations. At times, when the measurements of electric field are of good quality, it is possible to determine the sense of the wave vector without the $180^{\circ}$ ambiguity.

The data are from $C 1, C 2$ and $C 4$ spacecraft with the waves, the electrons and the magnetic fields measured by the same spacecraft. However, the ion moments from $C 2$ and $C 4$ are not available for time intervals in our sample (Table 1). In these time cases, we first compare the $B$-field data of $C 1$ and $C 2$ to see whether the two spacecraft were close enough to each other in order to observe the same regions in space. If this is true, then we use $C 1$ ion moments for calculating plasma parameters, such as electron gyrofrequency, etc. Table 1 shows information on the spacecraft that provided the data for each time interval.

\subsection{Case Study}

In this section we describe one case study in order to explain our methodology. We surveyed the data of the Cluster mission during the years 2001-2010. We searched for times when the Cluster was in the pristine SW, meaning that the satellites were not in the Earth's foreshock, nor was the SW perturbed by transient structures, such as stream interaction regions (SIRs) or interplanetary coronal mass ejections (ICMEs).

We use STAFF data sets in order to search for elliptically polarized, right-hand fluctuations, that propagate at small angles with respect to the background IMF. Figure 1 shows Cluster 1 observations from 11:40 UT to 12:15 UT on 18 April 2004. The top four panels show the IMF magnitude and GSE components in units of nanoTesla (nT), the SW number density $\left(\mathrm{cm}^{-3}\right)$ and the SW velocity $\left(\mathrm{km} \mathrm{s}^{-1}\right)$. The fourth panel shows the electric field dynamic spectrum from WHISPER. The lower four panels show the STAFF data: the dynamic spectrum of total energy of magnetic field fluctuations $B_{\text {SUM }}$, ellipticity $(+1$ 


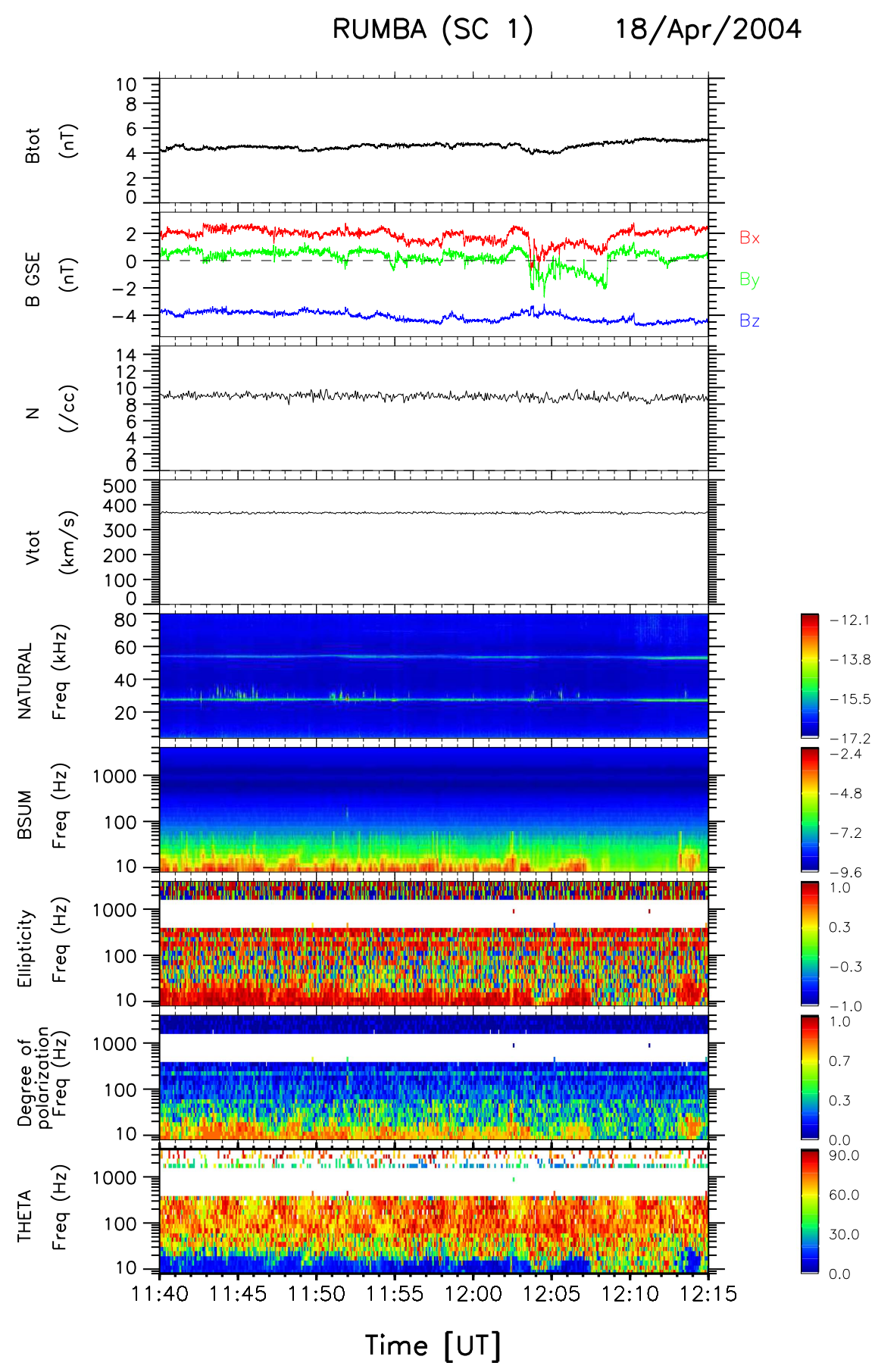

Figure 1. An example of whistler waves on 2004 April 18 observed by the Cluster 1 spacecraft. The panels show (from top to bottom): magnetic field magnitude and components in GSE coordinates (in units of $\mathrm{nT}$ ), the solar wind number density $\left(\mathrm{cm}^{-3}\right)$, and the solar wind velocity $\left(\mathrm{km} \mathrm{s}^{-1}\right)$. The fourth panel exhibits the electric field dynamic spectrum from the WHISPER. The lower four panels exhibit (from top to bottom): the dynamic spectrum of total energy of magnetic field fluctuations $B_{\text {SUM }}$, ellipticity $(+1$ for right-hand and -1 for left-hand polarized fluctuations $)$, degree of polarization $(0=$ linear, $1=$ circular $)$ and the angle of propagation (between the wave vector $k$ and the IMF, $\theta_{k B}$ ).

for right-hand and -1 for left-hand polarized fluctuations), degree of polarization $(0=$ linear, $1=$ circular $)$ and the angle of propagation (between the wave vector $k$ and the IMF, $\theta_{k B}$ ). During the presented time interval the spacecraft is in the pristine SW. The IMF and plasma properties are stable throughout the interval. The STAFF data show continuous $B$ field fluctuations in frequency range between $8 \mathrm{~Hz}$ and $\sim 20 \mathrm{~Hz}$ throughout the shown time intervals except between 12:07:30 UT and 12:13:00 UT. These fluctuations are righthanded, they are elliptically polarized and propagate with an angle $\theta_{k B}<30^{\circ}$, hence we classify them as whistler mode waves.

Figure 2 shows the average spectrum of magnetic fluctuations during the time interval from 11:53:00 UT to 12:03:00 UT. This spectrum is obtained by calculating the average power of fluctuations at each central frequency during that time. We plot the STAFF-SC part of the spectrum (below $\sim 12.5 \mathrm{~Hz}$ ) with a continuous purple line, while the asterisks represent the STAFF-SA data (above $\sim 12.5 \mathrm{~Hz}$ ). The spectrum consists of a continuous part, which belongs to background 


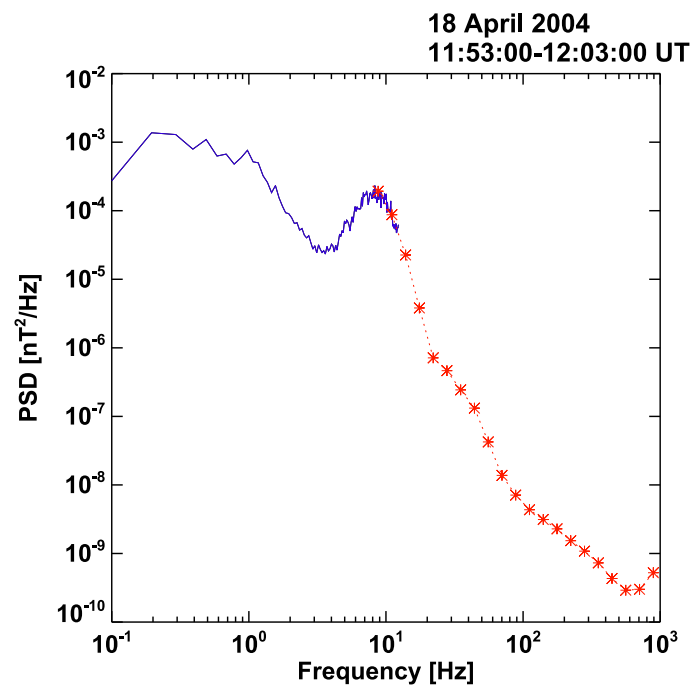

Figure 2. Whistler wave spectra on 2004 April 18. The STAFF-SC data are represented with a purple continuous line, while red asterisks are for the STAFF-SA data.

turbulence, and a distinct bump centered around $8 \mathrm{~Hz}$, which is due to the whistler mode waves.

Figure 3(a) shows the results of the minimum variance analysis (MVA, Sonnerup \& Sceible 1998) performed on the STAFF-SC data with $25 \mathrm{~s}^{-1}$ time resolution between 11:55:29.8 UT and 11:55:35.2 UT. It can be seen that the whistler waves are highly planar with the ratio of the intermediate and the minimum (Int/Min) variances of 58. In Figure 3(b) we show waveforms of these waves in the frame of eigenvectors obtained from the MVA. The panels (from top to bottom) show $B$-field profiles in the direction of the minimum, the medium, and the maximum variance.

Next, we use the PEACE PITCH_SPIN_DEFlux data sets in order to perform the measurements of the strahl width. These data sets contain the electron DEF as a function of the pitch angle (between the particle's velocity vector and the IMF) at spin (4s) time resolution for different central energies. The $\mathrm{DEF}$ is a product of the differential particle flux (DF) times the particle energy. The DF measures the number of particles with energy $d E$ about $E$ with direction $d \boldsymbol{\Omega}$ about $\boldsymbol{\Omega}$ that passes through the unit area perpendicular to $\Omega$ per unit time. Its units are $1 /\left(\mathrm{cm}^{2} \mathrm{~s}\right.$ str eV), while the DEF is measured in units of $\mathrm{eV} /\left(\mathrm{cm}^{2} \mathrm{~s}\right.$ str $\left.\mathrm{eV}\right)$.

We calculate the average pitch angle distribution (DEF versus PA) during one minute time intervals at twelve central energies between $\sim 57$ and $\sim 676 \mathrm{eV}$. We fit the PA distributions with a Gaussian function described by Equation (2):

$$
f(\mathrm{PA}, w)=f_{\text {halo }}+f_{0, \text { strahl }} e^{-\left(\frac{\mathrm{PA}-\mathrm{PA}_{0}}{\sqrt{2} w}\right)^{2}} .
$$

Here, $f_{\text {halo }}$ represents the constant contribution of the halo component, while the second term approximates the strahl distribution. PA stands for pitch angle (angle between the particle's velocity vector and the background magnetic field) and $w$ represents the width of the strahl centered at $\mathrm{PA}_{0}$, which can have values of $0^{\circ}$ or $180^{\circ}$. The fitting was performed by using the IDL CURVEFIT function. This function uses a gradient-expansion algorithm in order to provide a nonlinear least squares fit to any function with an arbitrary number of arguments. We adapted the CURVEFIT function in order to obtain the best estimates of $f_{\text {halo }}, f_{0 \text {,strahl }}$ and $w$ and also of their errors.

Figure 4 shows two examples of PADs observed on 2004 April 18, which are separated by a few minutes. On both panels the black asterisks represent the PADs from the data, while red crosses and dotted lines represent the best fits. The time intervals, the central energies and the widths of the fits are shown on the panels. We can see that during the time interval, when the whistler mode waves were present (left), the fitted width of the PAD is $\sim 32^{\circ} .2$, while during the time when the whistlers were absent, it is $\sim 27^{\circ} .4$. This is a large difference and it is much larger than the estimated width errors, which are $\sim 1^{\circ}$. The latter value is typical for our set of PADs.

We should state here that we visually inspected all the PADs in our sample in order make sure that the halo and the strahl components were present. This is important, since most of our samples were observed during the slow SW $\left(V_{\mathrm{sw}} \lesssim 400 \mathrm{~km} \mathrm{~s}^{-1}\right)$, while the strahl component has been recognized to be a permanent feature only in high-speed SW stream (Rosenbauer et al. 1977; Feldman et al. 1978). However, Pilipp et al. (1987b) showed that the strahl can be observed also in the slow SW.

\section{STATISTICAL RESULTS}

\subsection{IMF and $S W$}

Here, we briefly discuss the properties of the SW and the IMF during time intervals in our samples. All the intervals were selected so that the Cluster spacecraft were located in the pristine SW, far from any discontinuities such as interplanetary shocks, they were not inside the Earth's foreshock, nor within any transient structures such as ICMEs and SIRs. Transient structures, such as SIRs, are associated with enhanced magnetic field magnitudes. As suprathermal strahl electrons propagate into such regions, their PA distributions become wider due to conservation of the electron magnetic moment and this could interfere with our study.

Another reason for avoiding transient structures and IP shocks is that due to enhanced $B$-field magnitudes associated to them, some of the strahl electrons may be subject to adiabatic mirroring and propagate sunward at some acute PAs (between $0^{\circ}$ and $90^{\circ}$ ). Such populations of suprathermal electrons are known as conics (e.g., Gosling et al. 2001). As these electrons approach the Sun, they are again reflected due to strong magnetic field there and form another population called shoulders (Gosling et al. 2001) at PAs that are complementary to those of the conics. Associated to conics and shoulders are also halo depletions which are centered at $90^{\circ} \mathrm{PA}$ (Gosling et al. 2001; Lavraud et al. 2010). Suprathermal conics and halo depletions were also observed inside ICMEs (Feldman et al. 1999; Gosling et al. 2002). Additionally, Kajdič et al. (2014) reported observations of $90^{\circ} \mathrm{PA}$ enhancements near many IP shocks. We avoid all these electron signatures and select time intervals without them.

Average observational properties of SW and IMF from our sample were very similar, regardless of whether whistler mode waves were present or not (see Figure 5). The observed IMF magnitudes ranged between 1 and $12 \mathrm{nT}$ with the most common value at around $5 \mathrm{nT}$. All whistler waves were observed during times of slow SW $\left(v \leqslant 500 \mathrm{~km} \mathrm{~s}^{-1}\right)$ when the thermal pressure $P_{\text {th }}$ was $<0.05 \mathrm{nPa}$. The plasma density displayed average value of $7.8 \mathrm{~cm}^{-3} \pm 3.2 \mathrm{~cm}^{-3}$. Finally, the 


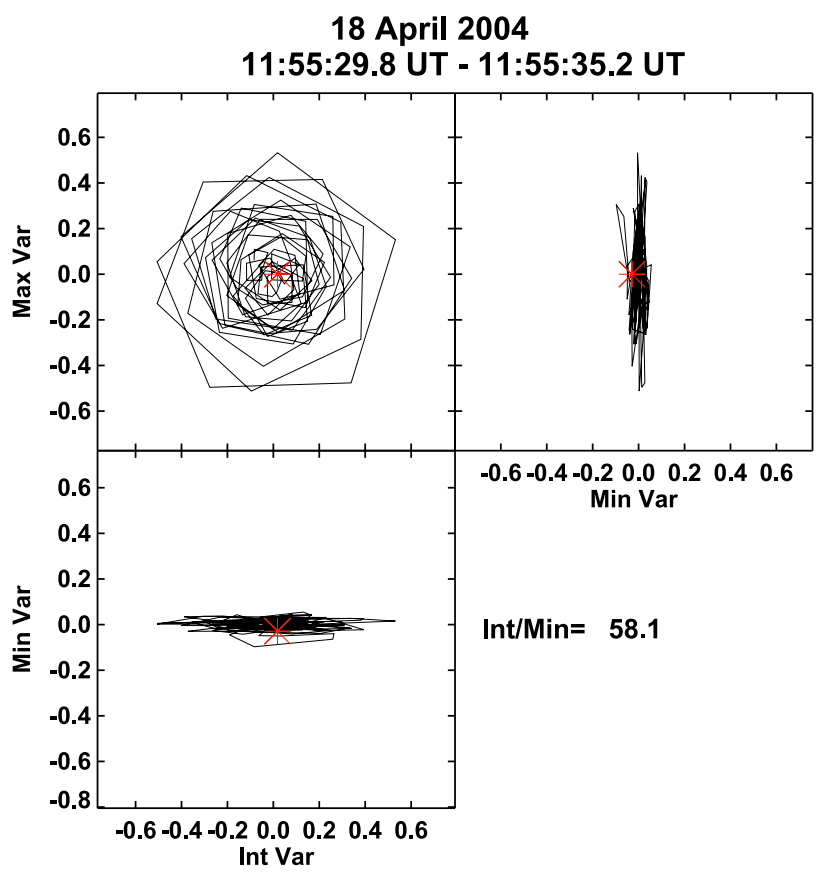

a)

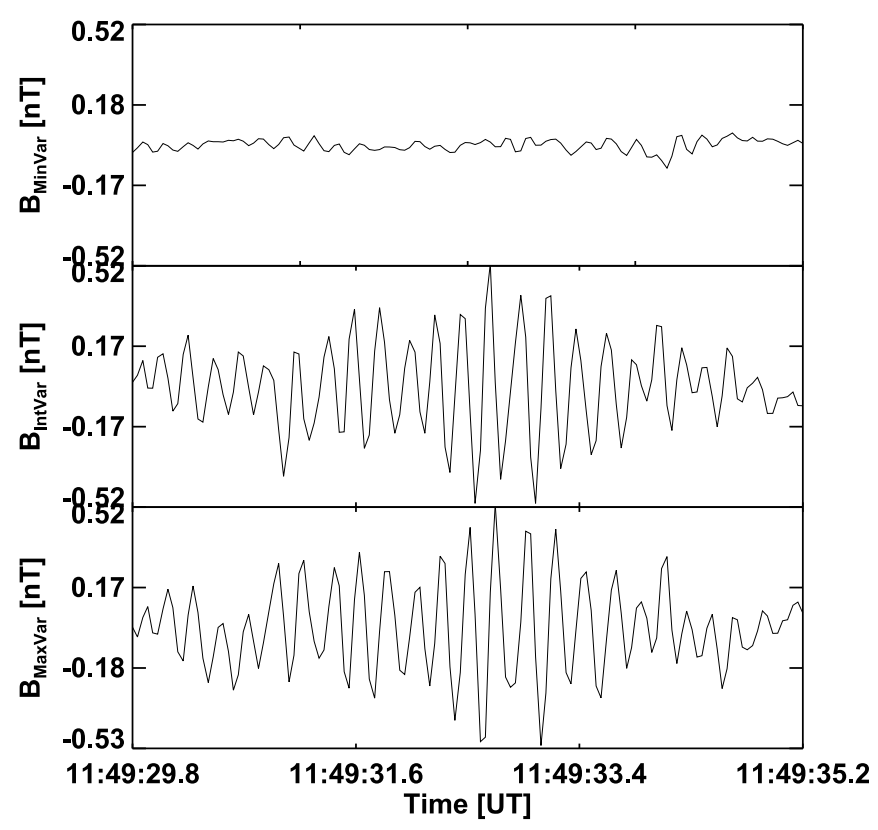

b)

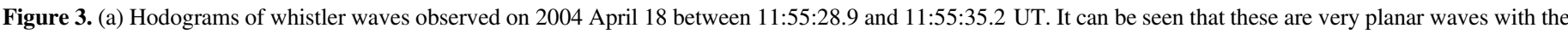

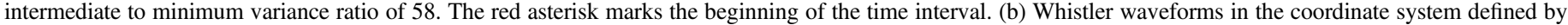

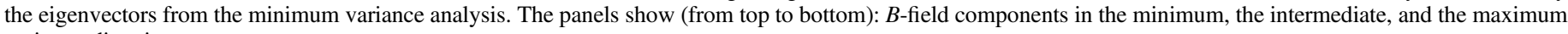
variance directions.

angle between the SW bulk velocity and the $\operatorname{IMF}\left(\theta_{B V}\right.$, not shown) was always above $45^{\circ}$. This has to do with the orbit of the Cluster mission. As explained by Alexandrova et al. (2012), when $\theta_{B V}$ is large there is more probability that the Cluster will not be magnetically connected to the Earth's bow shock (so it will be in pristine SW). For the whistler waves, this also means that since they propagate at small angles with respect to the background $B$-field, their frequencies in the spacecraft frame of reference will not be strongly Doppler shifted. The results on IMF and SW properties match well those reported by Lacombe et al. (2014) during their observations of the whistler mode waves.

\subsection{Whistler Wave Properties}

Figure 6 exhibits the distributions of the properties of the observed whistler waves. On panel (a) are shown their peak frequencies. These are all $\geqslant 5 \mathrm{~Hz}$ and tend to be less than $50 \mathrm{~Hz}$, although a few examples have been found at higher frequencies. We also show the whistler frequencies in units of electron gyrofrequency $\left(\Omega_{e}\right.$, panel (b) and the lower hybrid frequency $\left(\Omega_{L H}\right.$, panel (c). The observed $\omega / \Omega_{e}$ values range between $\sim 0.05$ and 0.3 with most of them being below 0.2 . The $\omega / \Omega_{L H}$ values are between $\sim 2$ and $\sim 7$.

The bottom three panels exhibit propagation properties of the observed whistler waves. The values shown are the averages of the propagation properties during the selected time intervals for central frequency, at which the whistler average wave spectra peak. In panel (d) we show the angle of propagation with respect to the IMF $\left(\theta_{k B}\right)$, which is between $5^{\circ}$ and $20^{\circ}$. On panel (e) we show the ellipticity, where positive values mean right-hand polarized waves and negative values mean left-hand polarization. In all of our cases the polarization is right-handed, as is should be for the whistler waves, with values between 0.7 and 1. Finally, panel (f) shows the degree of polarization (see Santolík et al. 2003, for details on polarization and ellipticity of waves from STAFF-SA data sets), where values close to 0 mean waves that are linearly polarized, while values close to 1 mean circularly polarization. All the waves exhibit the degree of polarization between 0.6 and 0.85 , which means that they are almost circularly polarized.

\subsection{Strahl Widths}

In this section we statistically compare the strahl widths for times when the whistler waves were present versus when they were absent.

In total we found 37 time intervals during which the whistler waves were present in the $B$-field spectra for at least one minute and all the required wave and particle data sets are available. We also selected 31 time intervals that were adjacent to, or at least very close to, the first 37 , but during which the whistler waves were not observed. This is a control sample. The reason that these intervals are fewer is because on some days the Cluster entered the pristine SW on three occasions and observed the whistlers on two of them. The remaining time interval was then used for a control sample. Both samples are required in order to compare the properties, such as the SW ion moments, the IMF strength, the strahl widths and the electron moments during times when the whistlers are present and when they are not. All the intervals are listed in Table 1.

The number of selected whistler intervals is not very large considering the long time period during which they were found. There are several reasons for this. The Cluster spacecraft do not spend much time in the pristine SW. We eliminated all the intervals when structures, such as ICME and 


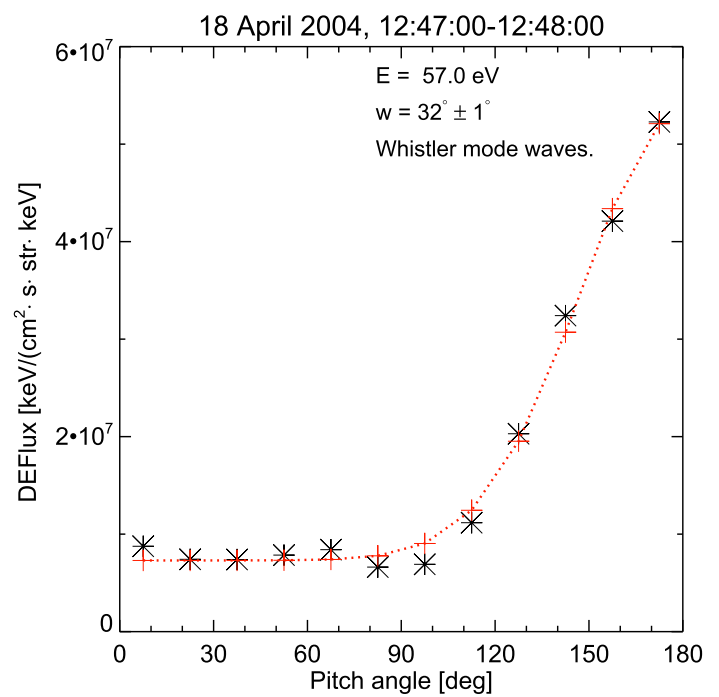

a)

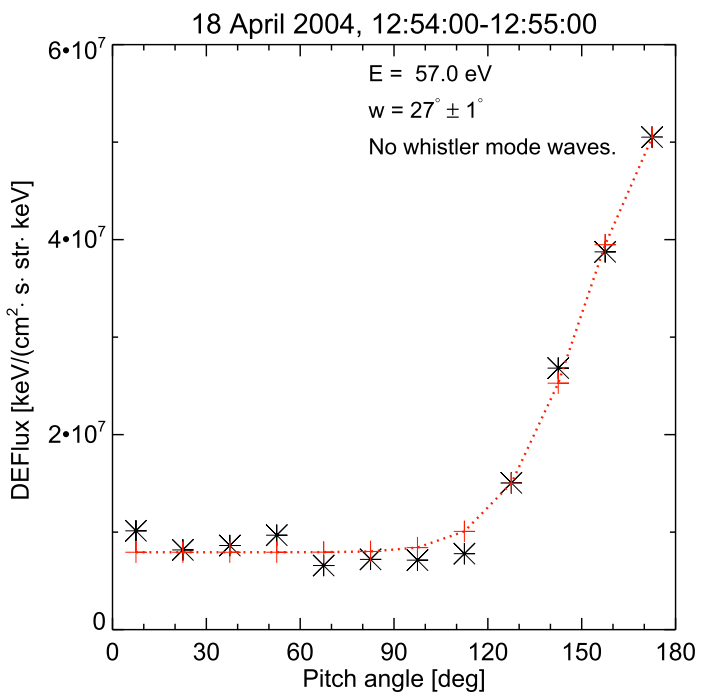

b)

Figure 4. Examples of PA distributions and the corresponding fits on 2004 April 18, during one minute time intervals, when the whistler mode waves were present (left), and when they were absent (right). Black asterisks show PADs from the data, while red crosses and dotted lines show the best fits.

SIRs, were present in the SW and also several different data sets (electron and ion data, magnetic field measurements, and STAFF and PEACE data sets) all had to be available for the purpose of this study. For comparison, Lacombe et al. (2014) report the presence of whistler waves in $\sim 10 \%$ of their selected data. However, these authors did not check for the presence of ICMEs or SIRs in the SW. Breneman et al. (2010), for example, found that intense whistler waves are most commonly found within the SIRs, close to IP shocks and near the heliospheric current sheet crossings.

We first divide each interval from Table 1 into consecutive one minute sub-intervals and calculate the average PADs during those times. Each PAD obtained this way is considered as one measurement in our sample. By doing this, we give more weight to longer time intervals and less to shorter ones. Next, we fit these average distributions with the function explained in Equation (2). We do this for twelve central energies for times with and without the whistlers and compare them. It should be noted that the total number of samples is different for different energies. In some cases the data was too noisy to allow the fitting. We visually inspect each fit in order to approve or reject it. The actual number of samples at each central energy is shown in Table 2. Finally we calculate the average values and the error of the mean of the strahl width at each electron energy.

Figure 7 exhibits the results of this comparison. On panel (a) we show the average strahl widths in presence (black asterisks) and absence (blue diamonds) of the whistler waves. We also plot the error bars indicating the error of the mean of each sample (the spread of the distribution). While on the lower $x$ axis we show electron energy in units of $\mathrm{eV}$, on the upper abscissa we show electron energy normalized to typical electron thermal energy $E_{T}$ in the SW. In order to calculate the latter we assumed a typical electron temperature in the SW to be $140,000 \mathrm{~K}$, which corresponds to $E_{T}=\frac{1}{2} k_{\mathrm{B}} T \sim 6 \mathrm{eV}\left(k_{\mathrm{B}}\right.$ is the Boltzmann constant). This electron temperature has been shown to be a very good approximation (Newbury 1996) independent of other SW parameters including the proton temperature (Feldman et al. 1977, pp. 351-382; Newbury 1996).

What can be seen in Figure 7(a) is that in the absence of the whistler waves (blue diamonds), the strahl width diminishes monotonically with increasing energy. The only exception is the width at $676 \mathrm{eV}\left(\sim 113 E_{T}\right)$, for which the number of one minute sub-intervals is smallest and the errors of the mean are largest. In the presence of the whistlers (black asterisks) the strahl width remains roughly constant between $\sim 111 \mathrm{eV}$ $\left(\sim 19 E_{T}\right)$ and $\sim 344 \mathrm{eV}\left(\sim 57 E_{T}\right)$. We should emphasize that this behavior is only observed on average. The behavior of the strahl width varies from case to case (not shown) and can be roughly constant or can diminish with increasing energy. In the past there have been some works that have reported different variations of the strahl width. Pilipp et al. (1987a, 1987b), Feldman et al. (1978, 1982), Hammond et al. (1996) and Fitzenreiter et al. (1998) reported diminishing widths of the strahl as a function of energy, similar to our case without whistler mode waves. On the other hand, the strahl width has been found to increase with energy in the presence of enhanced magnetic fluctuations, possibly whistler mode waves at frequencies $\lesssim 3 \mathrm{~Hz}$ (Pagel et al. 2007). Anderson et al. (2012) found that broadening or widening of the strahl occur with equal probability.

Figure 7(a) also shows that the average strahl widths in the presence of the whistlers are larger than when the whistlers are absent. This is true at all energies. The difference in the average strahl widths $(\Delta w)$ varies strongly with the electron energy (Figure 7(b)) but is always larger than the error bars, except at $676 \mathrm{eV}$. At $57 \mathrm{eV}\left(\sim 10 E_{T}\right)$ the difference in PA is $6^{\circ}$, it diminishes to $2^{\circ}$ between $89 \mathrm{eV}\left(\sim 15 E_{T}\right)$ and $140 \mathrm{eV}\left(\sim 23 E_{T}\right)$ and then it starts rising again. It reaches the maximum of $12^{\circ}$ at $344 \mathrm{eV}$.

Next, we show that the largest strahl widths occur preferentially when the differential energy flux, integrated over all pitch angles of the strahl, is more intense relative to the halo DEF. Figure 7(c) shows how the ratios of the strahl and the halo DEFs $\left(F_{\text {strahl }} / F_{\text {halo }}\right)$ vary with energy in cases when the 

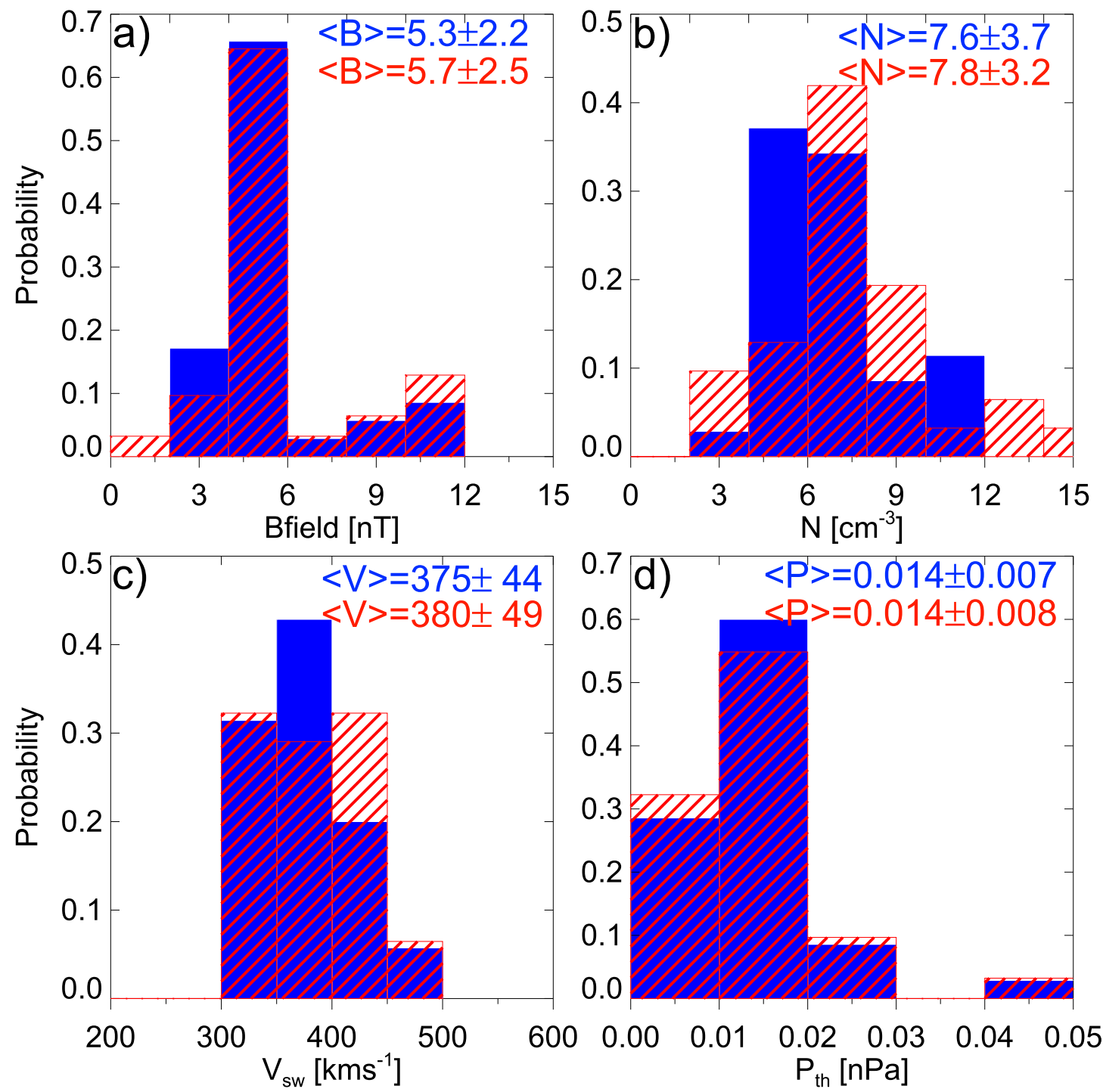

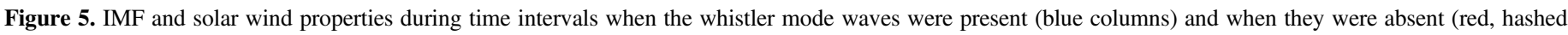

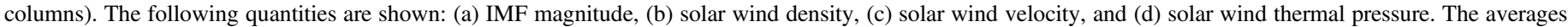
and standard deviations are also shown in the figure.

whistlers were present and when they were absent. In each individual case this ratio depends on central energies and energy ranges of the strahl and the halo components, so only average values of a larger sample of events can tell us whether this ratio is different when the whistler mode waves are present. We see in Figure 7(c) that regardless of the presence of whistlers the average $F_{\text {strahl }} / F_{\text {halo }}$ ratio increases for energies between 57 and $89 \mathrm{eV}$ and then it decreases for energies up to $175 \mathrm{eV}\left(\sim 29 E_{T}\right)$. After that the $F_{\text {strahl }} / F_{\text {halo }}$ ratio for times when there were no whistler waves remains roughly constant. During times when the whistlers were present however this ratio increases, reaching a peak at $\sim 280 \mathrm{eV}\left(\sim 47 E_{T}\right)$ and it decreases afterwards. The difference of $F_{\text {strahl }} / F_{\text {halo }}$ for times with and without the whistlers tends to be larger at energies at which the $\Delta w$ is also larger (compare with Figure 7(b)).

The error bars in Figure 7(c) are generally small and do not overlap. This means that the difference in $F_{\text {strahl }} / F_{\text {halo }}$ at most energies (with exceptions at 57 and $676 \mathrm{eV}$ ) is larger than the measurement errors.

\section{DISCUSSION}

In this work we perform a statistical study of the widths of suprathermal strahl in pristine SW at $1 \mathrm{AU}$ in the presence of narrow-band quasi-parallel $\left(k_{\|} \gg k_{\perp}\right)$ whistler waves observed within $[5,100] \mathrm{Hz}$ frequency range. The strahl widths during the time intervals with such whistler waves are compared to the strahl widths during times when the whistlers are absent. To our knowledge this is the first observational study that is trying to relate the narrow-band whistler waves in the pristine SW to the widening of the suprathermal electron strahl component.

In the past, the so called broad band whistler waves have been invoked in order to explain the strahl broadening. Pagel et al. (2007) report observations of broad strahl PA distributions during times of enhanced magnetic field fluctuations at $\leqslant 3 \mathrm{~Hz}$. During those intervals the strahl PA widths were broader than at earlier or later times. The authors used the Advanced Composition Explorer B-field measurements with time resolution of 3 vectors per second. They concluded that strahl PA broadening was due to broad-band magnetic field whistler fluctuations, although the authors did not explicitly 

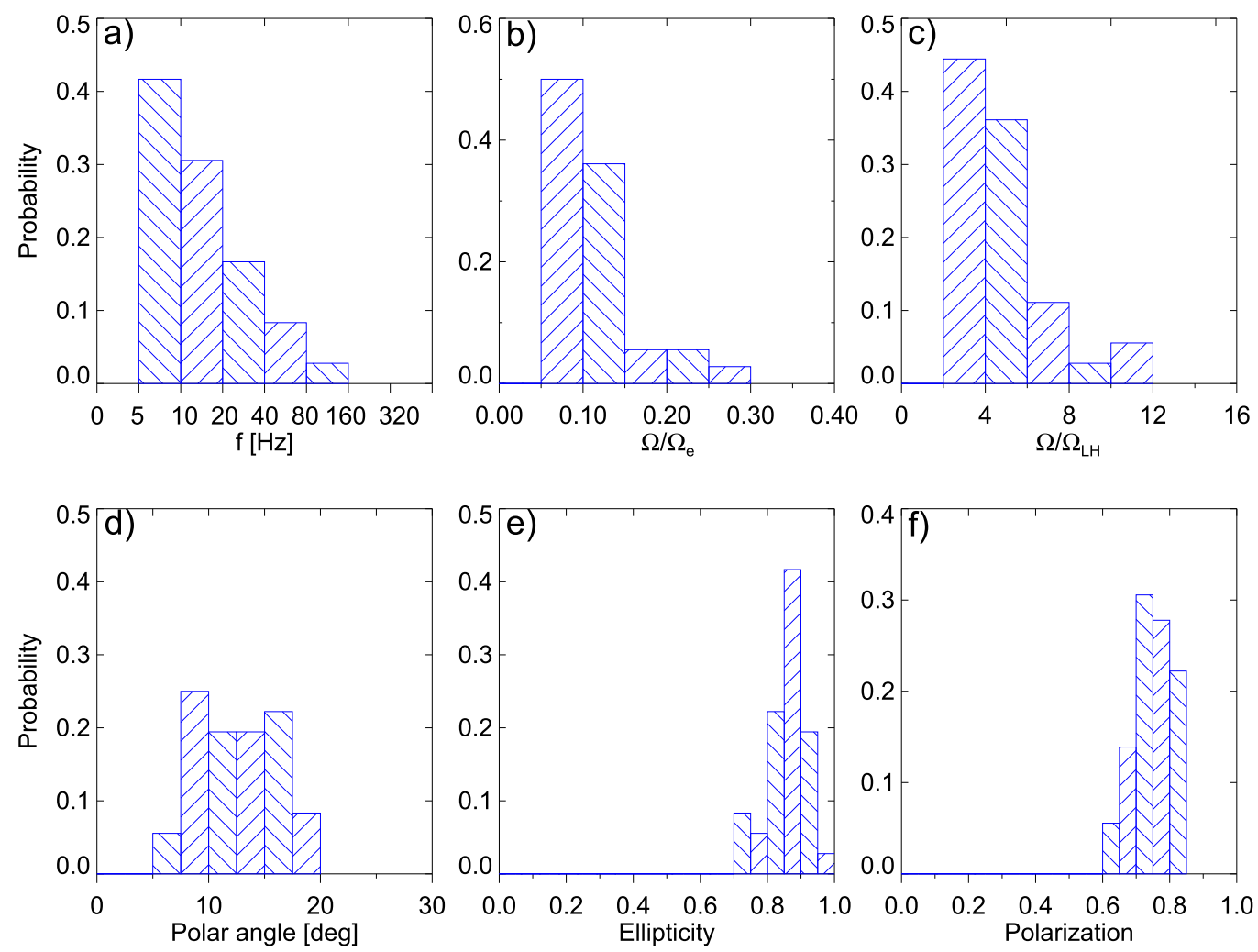

Figure 6. Distributions of observed whistler wave properties: (a) observed frequency, (b) whistler frequency normalized to the electron gyrofrequency, (c) whistler frequency normalized to lower hybrid frequency, (d) angle of propagation with respect to the background IMF, $\theta_{k B}$, (e) ellipticity, and (f) degree of polarization.

Table 2

Number of Samples of Suprathermal Electrons at Different Energies

\begin{tabular}{lcc}
\hline \hline $\begin{array}{l}\text { Central } \\
\text { Energy }(\mathrm{eV})\end{array}$ & $\begin{array}{c}\text { No. of Samples with } \\
\text { Whistlers }\end{array}$ & $\begin{array}{c}\text { No. of Samples without } \\
\text { Whistlers }\end{array}$ \\
\hline 676 & 48 & 22 \\
536 & 88 & 30 \\
430 & 114 & 38 \\
344 & 136 & 43 \\
276 & 163 & 66 \\
220 & 217 & 101 \\
175 & 275 & 123 \\
140 & 350 & 156 \\
111 & 398 & 197 \\
89 & 441 & 220 \\
71 & 469 & 233 \\
57 & 423 & 213 \\
\hline
\end{tabular}

show that the whistler mode waves, with broadband spectra, and with quasi-parallel wave vectors able to interact resonantly with electrons, were actually present during the observed time intervals. Numerical simulation results by Vocks et al. (2005) and theoretical considerations by Pierrard et al. (2011) have shown that if sunward propagating whistler waves with wave vectors parallel to the background magnetic field constituted the background turbulence, then their interaction with the suprathermal electrons would result in strahl broadening.

However, there is a problem when talking about the "broadband whistler waves" at sub-ion scales. Within this frequency range, whistler waves may exist and very often in the literature the authors call this range a "whistlers range". Whether such whistlers actually exist is still an open question. Observations show that the background turbulence in the SW at sub-ion scales are mainly transverse with the power in the direction perpendicular to the magnetic field $\left(\delta B_{\perp}^{2}\right)$ larger than the power in the parallel direction $\left(\delta B_{\|}^{2}\right)$ by as much as a factor of 20 (Chen et al. 2010). Both components (perpendicular and parallel) exhibit $k_{\perp}>k_{\|}$(Chen et al. 2010). The fact that wave vectors of the background turbulence are mainly perpendicular to the magnetic field was also shown by Mangeney et al. (2006) and Alexandrova et al. (2008). The general behavior of the SW background turbulence (with $k_{\perp} \gg k_{\|}$) at sub-ion scales was studied by Alexandrova et al. (2012). Although these observations are consistent with whistler mode waves, they are also consistent with kinetic Alfvén waves. However, even if they are whistler mode waves, in order for them to efficiently interact with parallel propagating strahl electrons, they are required to have wave vectors parallel to the background magnetic field. Hence, the observations of the electromagnetic turbulence in the SW are not favorable to the idea of broadband whistlers scattering the strahl electrons.

In this paper, we show that narrow-band whistler mode waves can efficiently interact with the strahl electrons. These waves are observed at around $0.1 f_{c e}$ (or within $[5,90] \mathrm{Hz}$ frequency range) in the SW and their wave vectors are almost parallel to the background $B$-field. Their durations had to be at least 1 minute in the spacecraft data in order to include them in our sample. The time intervals containing these waves have been chosen so the four Cluster spacecraft were in the pristine SW, so not inside the ICMEs, SIRs or the Earth's foreshock. We also excluded any intervals when the spacecraft were close to IP shocks or when electron distributions such as conics or shoulders were present. 


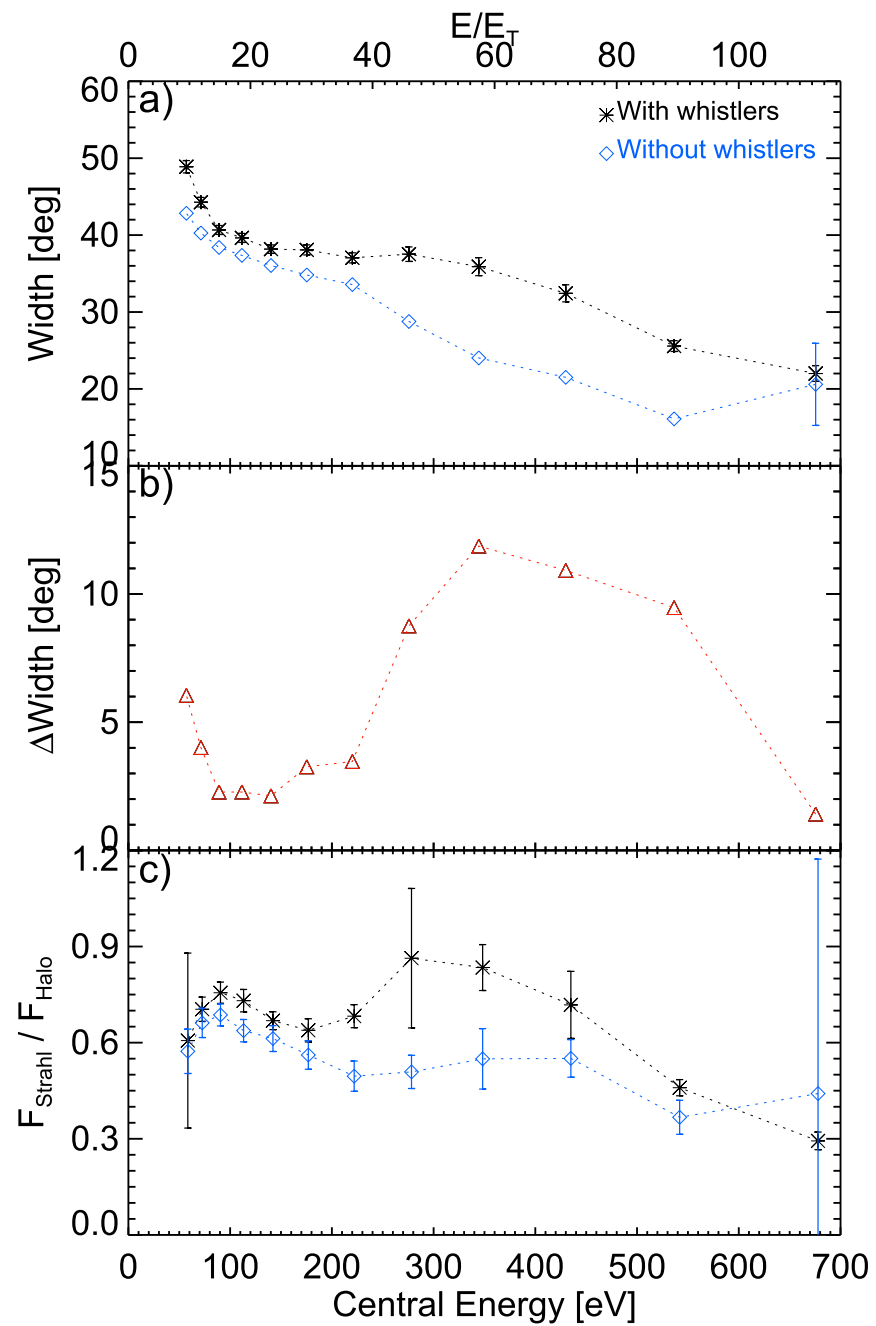

Figure 7. (a) Average strahl widths at times with the whistler waves (black asterisks) and during times when they were absent (blue diamonds). (b) Difference in strahl widths. (c) Strahl to halo flux ratios at times with and without the whistler waves.

We show that narrow-band whistlers modify the dependence of the strahl widths as a function of electron energy. The strahl broadening occurs at all energies, but its magnitude is different at different electron energies and ranges between $2^{\circ}$ and $12^{\circ}$ PA. Strahl widths no longer diminish monotonically as a function of the electron energy (as is the case in the absence of the narrow-band whistlers). On average, strahl widths diminish at energies below $\sim 111 \mathrm{eV}\left(\sim 19 E_{T}\right)$, then remain roughly constant and even slightly increase for $E \lesssim 276 \mathrm{eV}\left(\sim 46 E_{T}\right)$, and then they diminish again at higher energies. The largest difference between the average strahl width in the presence and absence of the whistler mode waves occurred at $E \sim 344 \mathrm{eV}$ $\left(\sim 57 E_{T}\right)$ and is equal to $\sim 12^{\circ} \mathrm{PA}$.

This energy dependence of the strahl width is different from what was reported in the past. Pilipp et al. (1987a, 1987b), Feldman et al. (1978, 1982), Hammond et al. (1996) and Fitzenreiter et al. (1998) studied strahl widths in the SW as a function of electron energy and concluded that the widths diminish with increasing energy of electrons. We also see in our Figure 7(a) that when the narrow-band whistler mode waves are not present, the strahl narrows monotonically with increasing electron energy. Pagel et al. (2007) reported increasing strahl widths as a function of electron energy in the presence of low frequency $(\leqslant 3 \mathrm{~Hz})$ magnetic field fluctuations. However, as we show here whistlers with parallel wave vectors are observed at $f>3 \mathrm{~Hz}$. Therefore, the role of whistlers in the observations of Pagel et al. (2007) is questionable.

The $F_{\text {strahl }} / F_{\text {halo }}$ ratio (Figure $7(\mathrm{c})$ ) at times without the whistlers shows very little dependence on the electron energy. At times when the whistlers were present this ratio is increased for energies between $\sim 220 \mathrm{eV}\left(\sim 37 E_{T}\right)$ and $\sim 536 \mathrm{eV}\left(\sim 89 E_{T}\right)$ and peaks at $\sim 280 \mathrm{eV}\left(\sim 47 E_{T}\right)$. In order to interact with strahl electrons in this energy range the whistler phase velocities need to be between $\sim 970 \mathrm{~km} \mathrm{~s}^{-1}$ and $\sim 1500 \mathrm{~km} \mathrm{~s}^{-1}$ (taking the whistler frequency $\omega=100 \mathrm{~Hz}, \Omega_{e}=1000 \mathrm{~Hz}$, and parallel propagation of the strahl electrons). The difference of $F_{\text {strahl }} /$ $F_{\text {halo }}$ ratios for times with and without the whistlers tends to be larger at energies at which the strahl width is also larger. This suggests that the more intense strahl relative to the core is related to wider strahls and to the presence of the whistler mode waves.

\section{CONCLUSIONS}

In this work we show that narrow-band whistler mode waves do interact with the strahl electrons. This interaction results in the broadening of the strahl PA width, which is different at different energies. The largest strahl broadening occurred at electron energy $E \sim 344 \mathrm{eV}\left(\sim 57 E_{T}\right)$ and was equal to $\sim 12^{\circ}$ PA. The dependence of strahl width as a function of energy is modified in the presence of the whistlers since the width no longer decreases monotonically with increasing energy as is observed in the absence of quasi-parallel propagating whistler waves. During times when the narrow-band whistlers are present, the ratio of strahl to halo fluxes $F_{\text {strahl }} / F_{\text {halo }}$ is also increased. The more intense strahl relative to the core is related to larger strahl widths and to the presence of the whistler mode waves.

The question arises how much the whistler mode waves in the interplanetary space contribute to strahl scattering in order to account for the formation of the halo component. Our study is performed with the Cluster data at $1 \mathrm{AU}$ and it does not show the accumulative effect that such interactions could have along the entire electron's trajectory from Sun to 1 AU. However, the broadening of strahl by up to $12^{\circ}$ PA suggests that it is plausible that narrow-band whistler waves contribute importantly to the overall broadening of the suprathermal electron strahl.

The authors are grateful to the Cluster Science Archive teams (ESA) and the CL/CLWeb team (IRAP) for the easy access to the Cluster data; to O. Santolik for the PRASSADCO program which gives the polarization and propagation properties of the magnetic fluctuations measured by STAFF-SA, and to $\mathrm{P}$. Robert for a polarization program of waveform data, STAFF-SC, and FGM. The authors also acknowledge the Cluster teams: STAFF (PIs P. Canu and N. CornilleauWehrlin), FGM (PIs C. Carr, A. Balogh and E. Lucek); CIS (PIs H. Rème and I. Dandouras); PEACE (PI A. Fazakerley). The CLUSTER STAFF instrument has been developed and operated with the support of CNES and CNRS. PK's work was supported by the PAPIIT grant IA104416.

Facility: CSA. 


\section{REFERENCES}

Alexandrova, O., Chen, C. H. K., Sorriso-Valvo, L., Horbury, T. S., \& Bale, S. D. 2013, SSRv, 178, 101

Alexandrova, O., Lacombe, C., \& Mangeney, A. 2008, AnGeo, 26, 3585

Alexandrova, O., Lacombe, C., Mangeney, A., Grappin, R., \& Maksimovic, M. 2012, ApJ, 760, 121

Anderson, B. R., Skoug, R. M., Steinberg, J. T., \& McComas, D. J. 2012, JGR, 117, A04107

Balogh, A., Carr, C. M., Acuña, M. H., et al. 2001, AnGeo, 19, 1207

Breneman, A., Cattell, C., Schreiner, S., et al. 2010, JGR, 115, A08104

Bruno, R., \& Carbone, V. 2013, LRSP, 2, 4

Chen, C. H. K., Horbury, T. S., Schekochihin, A. A., et al. 2010, PhRvL, 104, 255002

Cornilleau-Wehrlin, N., Chanteur, G., Perraut, S., et al. 2003, AnGeo, 21, 437

Cornilleau-Wehrlin, N., Chauveau, P., Louis, S., et al. 1997, SSRv, 79, 107

Décréau, P. M. E., Fergeau, P., Krannosels'kikh, V., et al. 1997, SSRv, 79, 157

Denskat, K. U., Beinroth, H. J., \& Neubauer, F. M. 1983, ZGeo, 54, 60

Fairfield, D. H., \& Scudder, J. D. 1985, JGR, 90, 4055

Feldman, W. C., Asbridge, J. R., Bame, S. J., \& Gosling, J. T. 1982, JGR, 87,7355

Feldman, W. C., Asbridge, J. R., Bame, S. J., Gosling, J. T., \& Lemons, D. S. 1978, JGR, 83, 5285

Feldman, W. C., Asbridge, J. R., Bame, S. J., \& Goslingn, J. T. 1977, in The Solar Output and Its Variation, ed. O. R. White (Boulder, CO: Colorado Univ. Press)

Feldman, W. C., Asbridge, J. R., Bame, S. J., Montgomery, M. D., \& Gary, S. P. 1975, JGR, 80, 4181

Feldman, W. C., Skoug, R. M., Gosling, J. T., McComas, D. J., \& Tokar, R. L. 1999, GeoRL, 26, 2613

Fitzenreiter, R. J., Ogilvie, K. W., Chornay, D. J., \& Keller, J. 1998, GeoRL, 25,249

Ghosh, S., Siregar, E., Roberts, D. A., \& Goldstein, M. L. 1996, JGR, 101,2493

Gosling, J. T., Skoug, R. M., \& Feldman, W. C. 2001, GeoRL, 28, 4155

Gosling, J. T., Skoug, R. M., \& Feldman, W. C. 2002, GeoRL, 29, 1573

Gurgiolo, C., Goldstein, M. L., Viñas, A. F., \& Fazakerley, A. N. 2012, AnGeo, 30, 163

Gurnett, D. A., \& Bhattacharjee, A. 2005, Introduction to Plasma Physics with Space and Laboratory Applications (Cambridge: Cambridge Univ. Press)

Gustafsson, G., Bostrom, R., Holback, B., et al. 1997, SSRv, 79, 137

Hammond, C. M., Feldman, W. C., McComas, D. J., Phillips, J. L., \& Forsyth, R. J. 1996, A\&A, 316, 350

Johnstone, A. D., Alsop, C., Burge, S., et al. 1997, SSRv, 79, 351

Kajdič, P., Lavraud, B., Zaslavsky, A., et al. 2014, JGR, 119, 7038
Lacombe, C., Alexandrova, O., Matteini, L., et al. 2014, ApJ, 796, 5

Lavraud, B., Optiz, A., Gosling, J. T., et al. 2010, AnGeo, 28, 233

Lin, R. P. 1998, SSRv, 86, 61

Maksimovic, M., Pierrard, V., \& Riley, P. 1997, GeoRL, 24, 1151

Maksimovic, M., Zouganelis, I., Chaufray, J.-Y., et al. 2005, JGR, 110, A09104

Mangeney, A., Lacombe, C., Maksimovic, M., et al. 2006, AnGeo, 24,3507

Montgomery, D. M., Bame, S. J., \& Hundhauses, A. J. 1968, JGR, 73, 4999

Newbury, J. A. 1996, Eos, Trans. Am. Geophys. Union, 77, 471

Ogilvie, K. W., Fitzenreiter, R., \& Desch, M. 2000, JGR, 105, 27277

Owens, M. J., Crooker, N. U., \& Schwadron, N. A. 2008, JGR, 113, A11104

Pagel, C., Gary, S. P., de Koning, C. A., Skoug, R. M., \& Steinberg, J. T. 2007, JGR, 112, A04103

Pavan, J., Viñas, A. F., Yoon, P. H., Ziebell, L. F., \& Gaelzer, R. 2013, ApJL, 769, L30

Phillips, J. L., \& Gosling, J. T. 1990, JGR, 95, 4217

Pierrard, V., Lazar, M., \& Schlickeiser, R. 2011, SoPh, 269, 421

Pierrard, V., Maksimovic, M., \& Lemaire, J. 1999, JGR, 104, 17021

Pilipp, W. G., Miggenrieder, H., Montgomery, M. D., et al. 1987a, JGR, 92, 1075

Pilipp, W. G., Miggenrieder, H., Muhlhauser, K.-H., et al. 1987b, JGR, 92, 1103

Rème, H., Aoustin, C., Bosqued, J. M., et al. 2001, AnGeo, 19, 1303

Roberts, O. W., Li, X., \& Li, B. 2013, ApJ, 769, 58

Rosenbauer, H., Miggenrieder, H., Montgomery, M., \& Schwenn, R. 1976, in Physics of Solar Planetary Environments, ed. D. J. Williams (Washington, DC: AGU), 319

Rosenbauer, H., Schwenn, R., Marsch, E., et al. 1977, J. Geophys, 42, 561

Sahraoui, F., Goldstein, M. L., Belmont, G., Canu, P., \& Rezeau, L. 2010, PhRvL, 105, 131101

Saito, S., \& Gary, S. P. 2007, JGR, 112, A06116

Santolík, O., Parrot, M., \& Lefeuvre, F. 2003, RaSc, 38, 1010

Scudder, J. D., \& Olbert, S. 1979, JGR, 84, 2755

Smith, C. W., Hamilton, K., Vasquez, B. J., \& Leamon, R. J. 2006a, ApJL, 645, L85

Sonnerup, B. U. O., \& Sceible, M. 1998, in Analysis Methods for MultiSpacecraft Data, ISSI Scientific Report SR-001, ed. G. Paschman \& P. W. Daly (Paris: ESA), 185

Stawicki, O., Gary, S. P., \& Li, H. 2001, JGR, 106, 8273

Štverák, Š., Maksimovic, M., Trávníček, P. M., et al. 2009, JGR, 114, 5104

Vocks, C. 2012, SSRv, 172, 303

Vocks, C., \& Mann, G. 2003, ApJ, 593, 1134

Vocks, C., Mann, G., \& Rausche, G. 2008, A\&A, 480, 527

Vocks, C., Salem, C., Lin, R. P., \& Mann, G. 2005, ApJ, 627, 540 